$$
\text { "daroczy" — 2003/5/19 — 23:07 — page 35 — \#1 }
$$

\title{
Gaussian iteration of mean values and the existence of $\sqrt{2}$
}

\author{
ZOLTÁN DARÓCZY
}

\begin{abstract}
We propose a method for proving the existence of $\sqrt{2}$ and finding its approximate value in secondary education.

Key words and phrases: rational numbers, irrational numbers, number line, continuity axiom, Gaussian iteration, inequalities for means, approximate value of $\sqrt{2}$.

ZDM Subject Classification: Mathematics Didactics Database F44, F54, F55, G90, H34, I35, N55.
\end{abstract}

1. In the secondary education the proof of $\sqrt{2}$ being an irrational number is well-known. Although the truth is that the proof does not assert the existence of $\sqrt{2}$. Therefore it does not even assert that there exist irrational numbers, not even $\sqrt{2}$. If we assume that the set of rational numbers is known then the statement in question can be formulated in the following way.

THEOREM 1. There is not any rational number whose square is 2.

So the problem is how to demonstrate the existence of $\sqrt{2}$ by a quick determination of approximate values in the secondary education.

2. According to my memories during the secondary education the existence of a length (positive number) whose square is 2 was proved geometrically and it Supported by FKFP 0590/1999 and by OTKA T043080.

Copyright (c) 2003 by University of Debrecen 


$$
\text { "daroczy" — 2003/5/19 — 23:07 — page 36 — \#2 }
$$

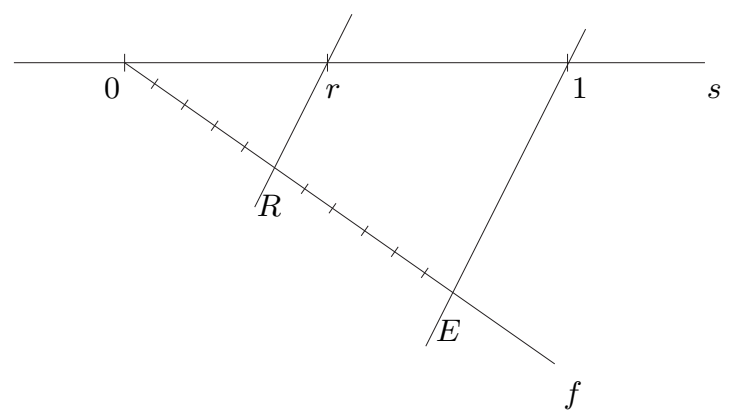

Figure 1

was called $\sqrt{2}$. Consider the number line s in the plane $S$, in which the points 0 and 1 are marked and the point 0 precedes 1 (Figure 1 ).

Then let $0<r=\frac{n}{m}<1$ be an arbitrary rational number where $n$ and $m$ are natural numbers. Draw a ray $f$ from the point 0 in the plane $S$ which forms an acute angle with $s$. Measuring some distance $d>0 m$ and $n$ times on $f$ (starting from the point 0$)$ we obtain the points $E$ and $R(m=11, n=5$ in Figure 1). Connect $E$ and 1 by a line and draw a straight line parallel to this through $R$. This parallel line intersects the line $s$ at $r$ which will be identified by the rational number $r=\frac{n}{m}$. In this way, by a Euclidean construction, we can assign points on the number line to any rational number, because each rational number can be uniquely written as $n+r$ where $n$ is an integer $(n \in \mathbb{Z})$ and $r$ is a rational number in $[0,1[$. Thus we obtain points of all rational coordinates on the number line $s$.

3. If we draw a perpendicular $m$ at the point 1 to the line $s$ in the plane $S$ and we measure the unit distance $\overline{01}$ on $m$ starting from the point 1 then we obtain the point $I$ (Figure 2).

Then by the Pythagorean theorem for the right-angled triangle 01I:

$$
c^{2}=1^{2}+1^{2}=2
$$

where $c$ is the length of the hypotenuse OI. That is, $c$ is a positive length whose square is 2. By a Euclidean construction, this distance $c$ can be measured online $s$ and we will obtain the point $c=\sqrt{2}$ on $s$ (Figure 2). A point like this exists on line $s$ and for its coordinate $c^{2}=2$ holds. Hence we can state that $c=\sqrt{2}$. On the other hand, there is a "hole" on the number line $s$ at point $c$ in the sense 


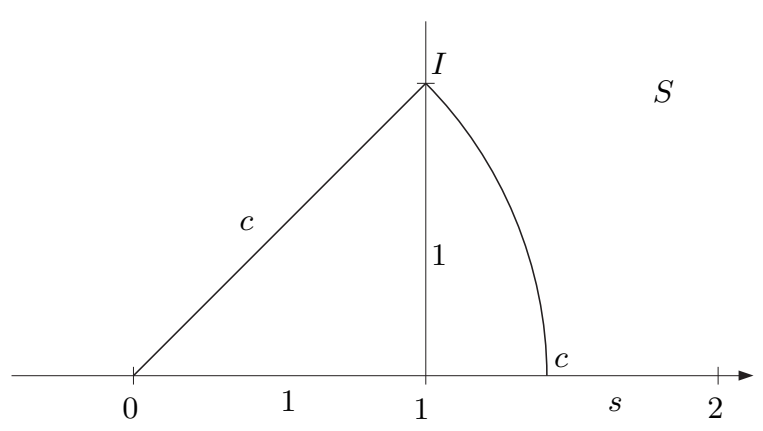

Figure 2

that in the construction described in Section 2, by virtue of the theorem 1 at that point there can not be a rational point. At the same time $c=\sqrt{2}$ is an existing real length (that is, a "real" number), which exists according to the facts mentioned above.

4. The above reasoning does not bring us closer to the concept of real numbers. Namely, according to Sections 2 and 3 only the constructible points can be assigned to points of the number line $s$. But it is not so in the case of a point $P$ for which $\overline{O P}=\pi$ (as $\pi$ is not a constructible distance). Therefore there are other "holes" on the number line. To solve the problem we can not evade the continuity axiom used by Hilbert which is, as far as I know, ignored in the secondary education (Hilbert [5]).

5. The concept of real numbers is treated in a deductive way at universities. The set $\mathbb{R}$ of real numbers is an ordered complete field in which we assign sets of natural numbers $(\mathbb{N})$, integers $(\mathbb{Z})$ and rational numbers $(\mathbb{Q})$. It is easy to show that $\mathbb{Q}$ is an ordered field therefore the assumption of the axiom of completeness draws a distinction between $\mathbb{Q}$ and $\mathbb{R}$. We know it well that several equivalent axioms can be given for the concept of completeness. The following two statements remain always true in $\mathbb{R}$ (they are axioms or consequence of axioms) (see Ebbinghaus [3], Stromberg [6]).

(a) Archimedean order: the set of natural numbers $\mathbb{N}$ is not bounded above (from which it follows that

$$
\left.\lim _{n \rightarrow \infty} \frac{1}{n}=0\right)
$$




$$
\text { "daroczy" — 2003/5/19 — 23:07 — page 38 — \#4 }
$$

(b) Cantor's theorem (axiom): if $I_{n}:=\left[a_{n}, b_{n}\right]\left(a_{n}<b_{n} ; a_{n}, b_{n} \in \mathbb{R}, n \in \mathbb{N}\right)$ is a sequence of closed intervals such that $I_{n+1} \subseteq I_{n}$ holds for every $n \in \mathbb{N}$ then

$$
\bigcap_{n=1}^{\infty} I_{n} \neq \emptyset \text {. }
$$

Additionally, it is also true that if in $(b)$

$$
\lim _{n \rightarrow \infty}\left(b_{n}-a_{n}\right)=0
$$

then the set

$$
\bigcap_{n=1}^{\infty} I_{n}
$$

has only one member, that is, there exists one and only one real number $x \in \mathbb{R}$ such that $x \in I_{n}$ for every $n \in \mathbb{N}$. In this case this sequence $I_{n}$ is usually called a nested sequence of intervals that has a unique kernel $x$. If we represent data mentioned above on the number line then we arrive at the continuity axiom used by Hilbert. If in $(b)$ for $I_{n}=\left[a_{n}, b_{n}\right]$ it is also true that $a_{n}, b_{n}$ are rational numbers and

$$
\lim _{n \rightarrow \infty}\left(b_{n}-a_{n}\right)=0
$$

then there exists exactly one point $x$ on the number line such that it is in each $I_{n}$ (Figure 3).

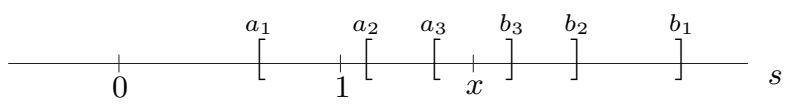

Figure 3

6. Gauss was 14 years old when he started to treat the following problem (Gauss [4]). Let us fix $0<x<y$ and $a_{1}:=x \quad b_{1}:=y$. Then according to the inequality between the arithmetical mean and the geometric mean

$$
x<\sqrt{x y}<\frac{x+y}{2}<y .
$$

Thus, by the notation

$$
a_{2}:=\sqrt{x y}=\sqrt{a_{1} b_{1}} \quad \text { and } \quad b_{2}:=\frac{x+y}{2}=\frac{a_{1}+b_{1}}{2}
$$




$$
\text { "daroczy" — 2003/5/19 — 23:07 — page 39 — \#5 }
$$

we have that

$$
a_{1}<a_{2}<b_{2}<b_{1}
$$

Going on with this procedure by the following iteration

$$
a_{n+1}=\sqrt{a_{n} b_{n}}, b_{n+1}=\frac{a_{n}+b_{n}}{2} \quad(n=1,2,3, \ldots)
$$

with the notation $I_{n}=\left[a_{n}, b_{n}\right](n \in \mathbb{N})$ we obtain that $I_{n+1} \subset I_{n}$ and from $0<b_{n}-a_{n} \leq \frac{y-x}{2^{n-1}}(n=1,2,3, \ldots)$ it follows that

$$
\lim _{n \rightarrow \infty}\left(b_{n}-a_{n}\right)=0 .
$$

Hence

is a one-element set and

$$
\bigcap_{n=1}^{\infty} I_{n}=\{M\}
$$

$$
\lim _{n \rightarrow \infty} a_{n}=\lim _{n \rightarrow \infty} b_{n}=M
$$

$M$ is called the Gaussian arithmetical-geometric mean of $x$ and $y$ and denoted by

$$
M=A G(x, y)
$$

The basic problem of Gauss was how to determine the value of $A G(x, y)$. At the age of 19 he wrote his remarkable result in his diary that reveals

$$
A G(1, \sqrt{2}) L=\frac{\pi}{2}
$$

where

$$
L:=\int_{0}^{1} \frac{d t}{\sqrt{1-t^{4}}}
$$

is the lemniscate constant. Gauss' result was a starting point of the theory of elliptic integrals (see Gauss [4], Borwein-Borwein [1]).

7. Gauss' idea can be applied to other theorems relating to mean values. In the set of rational (or real) numbers it is easy to prove the inequality between the harmonic and arithmetical mean values.

Theorem 2. If $0<x<y$ then $x<\frac{2 x y}{x+y}<\frac{x+y}{2}<y$. 


$$
\text { "daroczy" — 2003/5/19 — 23:07 — page } 40 \text { — \#6 }
$$

Analogously to the problem treated in section 6 , let us define the following quantities by Gaussian iteration:

$$
\begin{gathered}
a_{1}:=x \quad b_{1}:=y \\
a_{n+1}:=\frac{2 a_{n} b_{n}}{a_{n}+b_{n}}, \quad b_{n+1}:=\frac{a_{n}+b_{n}}{2} \quad(n=1,2,3, \ldots) .
\end{gathered}
$$

Then it is obvious that $I_{n+1} \subset I_{n}(n \in \mathbb{N})$ where $I_{n}:=\left[a_{n}, b_{n}\right](n \in \mathbb{N})$ and since

$$
0<b_{n}-a_{n} \leq \frac{y-x}{2^{n-1}} \quad(n \in \mathbb{N})
$$

we get

$$
\lim _{n \rightarrow \infty}\left(b_{n}-a_{n}\right)=0 .
$$

Therefore by Cantor's theorem there exists one and only one real number $p>0$ such that

$$
\bigcap_{n=1}^{\infty} I_{n}=\{p\}
$$

and

$$
\lim _{n \rightarrow \infty} a_{n}=\lim _{n \rightarrow \infty} b_{n}=p
$$

On the other hand, it is easy to see that

$$
a_{n+1} b_{n+1}=a_{n} b_{n} \quad(n \in \mathbb{N}),
$$

from which it follows that

$$
a_{n+1} b_{n+1}=a_{n} b_{n}=a_{n-1} b_{n-1}=\cdots=x y
$$

that is, $p^{2}=x y$ holds. As $p>0$ we have $p=\sqrt{x y}$ and thus the Gaussian iteration yields the geometric mean value.

8. In the secondary education facts described in section 7 can be discussed with the initial values $x=1$ and $y=2$. Then $a_{1}=1, b_{1}=2$ and $a_{2}=\frac{2 a_{1} b_{1}}{a_{1}+b_{1}}=\frac{4}{3}$, $b_{2}=\frac{a_{1}+b_{1}}{2}=\frac{3}{2}$ are rational numbers from which by induction we can prove that $I_{n}=\left[a_{n}, b_{n}\right]$ is a sequence of closed intervals having rational endpoints. According to facts mentioned previously there exists only one $p \in] 1,2[$ such that $p^{2}=2$. This existing real number is called $\sqrt{2}$. As $a_{n}<\sqrt{2}<b_{n}$ for any $n$ the 


$$
\text { "daroczy" — 2003/5/19 — 23:07 — page } 41 \text { — \#7 }
$$

determination of $\sqrt{2}$ by iteration can be carried out in the following way.

$$
\begin{array}{ccc} 
& a_{n} & b_{n} \\
n=1 & 1 & 2 \\
n=2 & \frac{4}{3} & \frac{3}{2} \\
n=3 & \frac{24}{17} & \frac{17}{12} \\
n=4 & \frac{941664}{665857} & \frac{665857}{470832}
\end{array}
$$

Calculating $a_{n}, b_{n}$ for $n=4,5$ up to 12 digits we have

$$
\begin{array}{ll}
a_{4}=1.414211438475 & b_{4}=1.414215686275 \\
a_{5}=1.414213562372 & b_{5}=1.414213562375
\end{array}
$$

We can see that the digits at the first 5 decimal places in $a_{4}, b_{4}$ are the same, while in $a_{5}, b_{5}$ the digits at the first 11 decimal places agree (printed in bold). It demonstrates that the iteration is very fast (certainly this concept can not be treated in the secondary education).

9. As a closing-thought we can say that Theorems 1 and 2 can be considered as part of the curriculum in the secondary education. Through a delicate practice of an intermediate teacher it is easy to be believed that from facts in section 8 it follows that there exists such a real number whose square is 2 and with today's modern technical background the approximate value of it can be determined very quickly and with great precision. Certainly I do not state that all this can be taught with perfect mathematical precision in the secondary education. The really important thing is what my former grammar school teacher told me: "The teacher should always tell true mathematical statements which the students believe and understand easily. However the teacher should know where and why he or she cheated." During university education the existence of $\sqrt{a}(a>0)$ or that of $\sqrt{2}$ can be taught with full precision in the way mentioned above. The Gaussian iteration can be defined to other pairs of mean values and researches relating to these mathematical problems are being carried out even today (see Daróczy-Páles [2] and the references therein). 


\section{References}

[1] J. M. Borwein and P. B. Borwein, Pi and the AGM, John Wiley, New York, 1987.

[2] Z. Daróczy and Zs. Páles, Gauss-composition of means and the solution of the Matkowski-Sutô problem, Publ. Math. Debrecen 61 (2002), 157-218.

[3] Ebbinghaus et al., Zahlen, Springer Verlag, 1983.

[4] C. F. Gauss, Bestimmung der Anziehung eines elliptischen Ringen, Nach lass zur Theorie des arithmetisch-geometrischen Mittels und der Modulfunktion, Akad. Verlag. M.B.H. Leipzig, 1927.

[5] D. Hilbert, Grundlagen der Geometrie, 3. Aufl, Leipzig und Berlin, 1909.

[6] K. R. Stromberg, An introduction to classical real analysis, Wadsworth Int. G., 1981.

ZOLTÁN DARÓCZY

DEPARTMENT OF MATHEMATICS AND INFORMATICS

DEBRECEN UNIVERSITY

H-4010 DEBRECEN, P.O. BOX 12

HUNGARY

E-mail: daroczy@math.klte.hu

(Received October 2, 2002) 